\title{
Empatía y educación en la infancia: un estado actual de la cuestión
}

\author{
Fausto-Andrés Núñez ${ }^{1}$ \\ Leidy-Tatiana Porras-Cruz ${ }^{2}$ \\ Ruth-Nayibe Cárdenas-Soler ${ }^{3}$
}

Fecha de recepción: 16 de marzo de 2021

Fecha de aprobación: 09 de abril de 2021

\section{Resumen}

El creciente interés por la educación emocional en la infancia motivó este trabajo, que busca examinar los aportes de diversas investigaciones, a partir de una revisión sistemática de documentos indexados en Google Académico, Web of Science, Science Direct, Scielo y Dialnet. Se consideró un total de 204 documentos, los cuales se referencian en la construcción narrativa de este texto; sin embargo, con el ánimo de determinar su pertinencia y relevancia, se hizo uso de matrices de análisis de contenido documental, a partir de las cuales se dio prevalencia a 62 documentos (desde 1985 hasta 2021). Los resultados se presentan en 3 categorías de análisis, que a su vez guiaron la revisión: empatía y razonamiento moral, empatía y desarrollo infantil; empatía en el ámbito educativo. Dentro de las conclusiones, es posible afirmar que existe una tendencia a centrar esta clase de estudios en las conductas prosociales y disruptivas en el ámbito educativo, además de sus posibles

\footnotetext{
${ }^{1}$ M. Sc. (c) Institución Educativa Técnica Agropecuaria San Isidro de Boyacá (Tunja-Boyacá, Colombia). ORCID: 0000-0003-0017-6471

2 M. Sc. (c) Universidad Pedagógica y Tecnológica de Colombia (Tunja-Boyacá, Colombia). leidytatiana.porras@uptc.edu.co. ORCID: 0000-0002-5860-9842

3 Ph. D. Universidad Pedagógica y Tecnológica de Colombia (Tunja-Boyacá, Colombia). ruth.cardenas@uptc.edu.co. ORCID: $\underline{0000-0003-4997-4116}$
} 
acciones educativas; por otra parte, estos hallazgos permiten determinar futuras líneas de trabajo en torno a la educación emocional y la empatía en la infancia.

Palabras clave: educación de la primera infancia; empatía; escuela; infancia.

\title{
Empathy and Education in Infancy and Childhood: A Current State of the Art
}

\begin{abstract}
The growing interest in emotional education in childhood motivated this work, which seeks to examine the contributions of various investigations, based on a systematic review of documents indexed in Google Scholar, Web of Science, Science Direct, Scielo and Dialnet. A total of 204 documents were considered, which are referenced in the narrative construction of this text; However, with the aim of determining its relevance and relevance, documentary content analysis matrices were used, from which 72 documents were given prevalence (from 1985 to 2021). The results are presented in 3 categories of analysis, which in turn guided the review: empathy and moral reasoning, empathy and child development; empathy in the educational field. Within the conclusions, it is possible to affirm that there is a tendency to focus this kind of studies on prosocial and disruptive behaviors in the educational field, in addition to their possible educational actions; On the other hand, these findings allow us to determine future lines of work around emotional education and empathy in childhood.
\end{abstract}

Keywords: childhood; early childhood education; empathy; school.

\section{Para citar este artículo:}

Núñez, F.A., Porras-Cruz, L.T. \& Cárdenas-Soler, R. N. (2021). Empatía y educación en la infancia: un estado actual de la cuestión. Pensamiento y Acción, 31, 74-90.

Esta obra está bajo licencia internacional Creative Commons Reconocimiento 4.0 


\section{Introducción}

Diversos estudios a lo largo de la historia han definido la empatía como principio orientador para comprender procesos de adaptación, convivencia y conducta prosocial; constituyendo elementos integradores que permiten a los individuos entender el contexto propio y el de los demás (Cuff et al., 2016). Actualmente, existen varias conceptualizaciones del término 'empatía', por su carácter multidimensional (Montgomery et al., 2016). Son Chlopan et al. (1985) son los primeros en tratar de definir la capacidad para comprender mentalmente la posición del otro o si se trata de la asimilación de las emociones ajenas, análisis que cambiaría significativamente el sentido del término (Decety \& Yoder, 2016). De esta forma, el presente documento reúne las aproximaciones teóricas en relación con el desarrollo y los procesos educativos en la educación infantil, en los cuales se evidencia la creación de vínculos y lazos sociales. Este artículo es el resultado del análisis documental realizado en el marco de la investigación "La capacidad empática en la convivencia familiar. Un estudio de caso", para lo cual pretende dar cuenta de tendencias investigativas en torno al desarrollo de la empatía en la infancia y la educación infantil.

En primer lugar, se hace necesario conceptualizar el término empatía, teniendo en cuenta su origen en siglo XVIII, con Robert Vischer (citado en Davis, 1996), quien lo refiere como "sentirse dentro de", acudiendo a su raíz griega "sentir dentro". De igual forma, autores como Adam Smith, Lipps y Köhler (citados en Wispé, 1986) hablaron de la importancia de sentir compasión por los demás, asimilar las emociones ajenas o imitar ciertos comportamientos prosociales en las interacciones sociales, siendo esta definición es la que se utiliza en la actualidad, además de concebirla (la empatía) como la facultad que tiene el ser humano de compartir sus emociones y entender las respuestas emocionales de otros individuos ante una situación específica (Angulo et al., 2019). Aunque varios autores trabajaron el término y aportaron a su desarrollo, es a Wipse (1986) a quien se le atribuye principalmente la construcción del concepto. Por su parte, Davis (1996) complementa la definición, describiendo la empatía como la capacidad que se trasmite genéticamente, esto le permite configurarla como una capacidad innata; 
afirmación que se sostiene con base en la selección natural de Charles Darwin, teniendo en cuenta que reconocer las necesidades emocionales de los demás genera respuestas emocionales altruistas. En el mismo sentido, Pavarino et al. (2005) argumentan que habilidades como la observación, la atención, la escucha, el interés y la preocupación por el otro congregan la empatía (Javakhishvili \& Vazsonyi, 2021).

Aunque es difícil establecer un concepto único que encierre la empatía como significado, es posible integrar al concepto la frase 'ponerse en los zapatos del otro' o estar en la capacidad de 'estar dentro de la misma situación' que experimenta el otro, para comprender su posición y estar en la posibilidad de ayudar o solidarizarse con él. Esta visión relacional incluye la necesidad de hacer una lectura desde la perspectiva cognitiva, emocional y situacional, lo que indica que el concepto es esencialmente multidimensional. Hay que resaltar que en los últimos años el concepto de empatía ha sido estudiado bajo el término 'symhedonia' (simpatía por la buena fortuna del otro). Sin embargo, algunos estudios refieren la empatía como aquella respuesta afectiva ante el estado emocional de otra persona, denominada resonancia emocional (Eisenberg \& Eggum, 2009), que puede conllevar a la simpatía o angustia personal, encontrándose relación con lo planteado por Nickerson y Mele-Taylor (2014), y Shamay-Tsoory et al. (2009), al evidenciar la relación entre empatía positiva y negativa.

Por lo mencionado, la empatía se entiende y conceptualiza desde dos enfoques: cognitivo y afectivo, aspectos reconocidos por Belacchi y Farina (2012) apuntando que, además, se trata de conductas prosociales y que se encuentran explícitos en cuatro aspectos conceptuales del Test de Empatía Cognitiva y Afectiva propuesto por López-Pérez et al. (2008), los dos primeros relacionados específicamente con la empatía cognitiva-: 1) la adopción de perspectiva (capacidad de ponerse en el lugar de otra persona); 2) la comprensión (capacidad de comprender los estados emocionales de otros; 3 ) estrés empático (capacidad de compartir las emociones negativas de otra persona, sintonizando emocionalmente con ella); y 4) alegría empática (capacidad de compartir emociones positivas) (Davis, 1996; Eisenberg \& Strayer, 1987; Smetana \& Ball, 2019) en base a respuesta de experiencias 
emocionales de otra persona (Zhang \& Wang, 2019; Zahn-Waxler et al, 1990) y la capacidad para comprender lo que pasa por la mente de los demás y a su vez hacer referencia a su importancia, lo que le permite una integración al mundo social (Chopik et al. 2016), teoría sería apoyada por la teoría afectiva de la mente de autores como Shamay-Tsoory et al. (2005) y Nilsen y Valcke (2018).

\section{Método de investigación}

Este trabajo surge de un análisis documental, el cual se caracteriza por ser una metodología que posibilita abordar rigurosamente un tema a partir de investigaciones publicadas en revistas científicas (Díaz \& Vega-Valdés, 2003). El objetivo general del trabajo era analizar las tendencias investigativas de la empatía, la infancia y el contexto escolar, de manera que se pudieran detectar sus principales aportes.

Se decidió realizar una revisión sistemática de documentos indexados en Web of Science, Science Direct, Scielo, Dialnet y Google Académico. Las palabras clave utilizadas en la búsqueda fueron: empatía, infancia y contexto escolar. Se detectaron e incluyeron 204 documentos en la matriz de análisis que incluían: artículos de investigación, revisiones, reflexiones, documentos publicados entre los años 1985 y 2021.

La búsqueda de los artículos se realizó en mediante el uso de palabras clave antes mencionadas y su correspondiente palabra en inglés incluyendo los operadores booleanos "and" y "or". Dentro de los criterios de elegibilidad, además de coincidir con las palabras clave, se consideró que se tratara de investigaciones realizadas entre los años 1985 y 2021 en las áreas de educación y psicología, que fueran textos completos, con preferencia en artículos de investigación y revisión.

Producto del ingreso de los 204 documentos a la matriz de análisis, se detectaron 141 documentos correspondientes a artículos en las áreas de educación y psicología y 62 de ellos publicados en fechas posteriores a 1986, momento en que aparece el concepto de empatía definido por Wispé (1986).

El proceso de categorización fue deductivo, con base en la lectura de los resúmenes

o las introducciones de los documentos, de forma que se establecieron los 
constructos: empatía y moral, empatía y desarrollo infantil, empatía en el ámbito educativo y empatía y conductas disruptivas.

Los criterios de exclusión fueron para los documentos que estuvieran fuera de la ventana de tiempo establecida, artículos publicados en ciencias de la salud, artículos restringidos por ser de acceso abierto, tesis y aquellos que no pertenecieran a la población infantil (Figura 1).

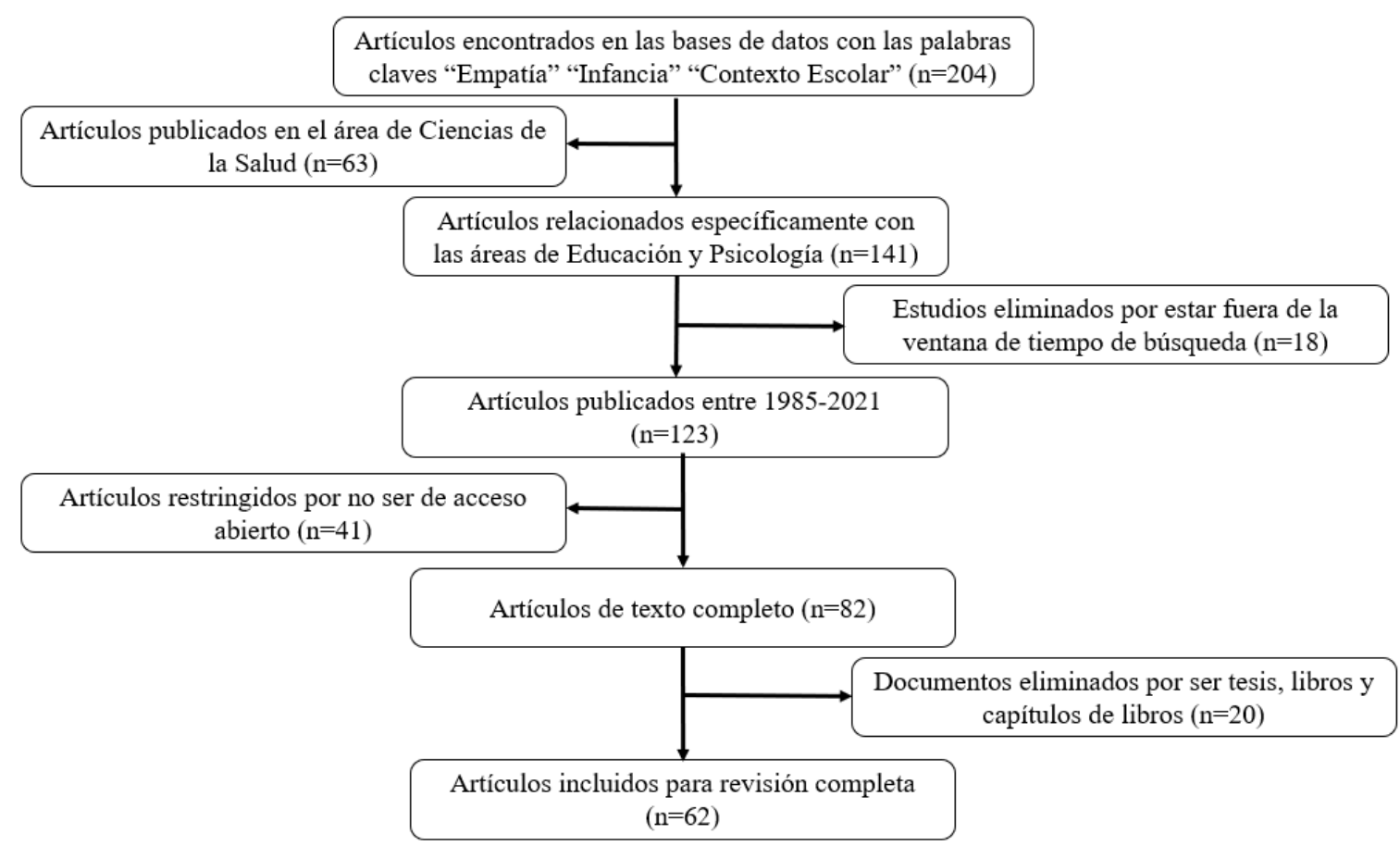

Fig. 1. Diagrama de búsqueda y selección de las fuentes analizadas. Los cuadros a la derecha e izquierda hacen parte de los criterios de exclusión para la selección de artículos.

Para la exploración inicial se construyó una matriz de análisis con los siguientes tópicos: año, título, autor, país, base de datos, tipo de documento y categoría. En el análisis del contenido se establecieron algunas unidades de análisis referente a la población de estudio y los temas más predominantes en el contexto escolar.

\section{Resultados}

En total se analizaron 79 artículos, de los cuales la mayoría fueron realizados en países como: Estados Unidos (27\%), España (11\%), Canadá (5\%), Argentina (5\%), 
Israel (4\%), Colombia (3\%) y Brasil (3\%), en los cuales se establecen algunas tendencias investigativas que se muestran a continuación.

\section{Tendencias investigativas de la empatía en la infancia}

A partir del análisis documental realizado se establecieron las siguientes tendencias investigativas en relación con la infancia y la empatía: educación y moral, empatía y desarrollo infantil, empatía en el ámbito educativo, y empatía y conductas disruptivas. Ahora bien, se detallarán las investigaciones y principales hallazgos en torno a cada uno de los tópicos señalados.

\section{Empatía y razonamiento moral}

Es importante resaltar que el desarrollo de la moral y la empatía en la infancia ha surgido con más ímpetu en los últimos años, con trabajos como los de Kim y Kochanska (2017) y Reese et al. (2007). Algunas investigaciones en esta línea han mostrado el desarrollo de la moral y su configuración en los primeros años de vida, siendo la primera infancia donde se generan relevantes avances en el desarrollo emocional y social (Bian et al., 2017). A este respecto, estudios han revelado que alrededor de los cuatro años, los niños pueden comprender las creencias sobre las experiencias emocionales de otros y que años más tarde, a esta habilidad se suma la teoría de la mente, consolidando los procesos de comprensión emocional (Miller, 2009; Misailidi \& Tsiara, 2021), y de moralidad en las acciones sobre los demás, por lo que se encuentra relación entre estos dos conceptos: teoría de la mente y moral (Smetana et al., 2012). Sin embargo, hay excepciones, porque otros estudios con niños no relacionan los juicios morales con las atribuciones emocionales, por ejemplo, el de Malti et al. (2010) quienes afirman que no se encuentran relacionados estos conceptos ya que definen por otros aspectos y la experiencia de culpa surge en la infancia, de forma que se establece el inicio del sistema interno de reglas y estándares morales, siendo este suceso motivación para evitar acciones inmorales. 


\section{Empatía y desarrollo infantil}

El estudio del desarrollo de la empatía en la infancia se configura bajo el análisis sobre las relaciones afectivas establecidas desde los primeros años de vida y su influencia en el desarrollo emocional de los menores, así como de los procesos cognitivos y emocionales que se tejen a nivel familiar (Nielsen et al., 2017). Lo anterior fue confirmando mediante el análisis que realiza De Minzi et al., (2011), quienes destacan que el desarrollo de la empatía se debe a factores genéticos y ambientales, ya que los procesos cognitivos y emocionales se aprenden por imitación, a pesar de la carga genética, lo cual determina unas bases importantes al interior de los sistemas familiares (Wong \& Yeung, 2019). Es decir que las relaciones parentales determinan no solo los rasgos físicos y conductuales, sino que involucran el desarrollo social, la personalidad, la proactividad, las alianzas sociales, el cooperativismo y los valores que identifican a las familias, siendo estas habilidades desarrolladas en los procesos de socialización primaria de niños (Decety et al., 2018; Levy et al., 2019). Así, se suman los procesos neurobiológicos, en la imitación que involucra a las neuronas espejo, donde hay que destacar que este desarrollo permite la formación de múltiples conexiones entre las estructuras corticales y subcorticales, permitiendo al sujeto emocionarse con otro, proceso que se puede evidenciar desde el nacimiento, cuando el niño, por ejemplo, responde a los estados emocionales de aflicción de otros, lo que significa aprender a sintonizarse emocionalmente con su cuidador y aprende a 'regular sus emociones', mediante el apego seguro expresado en comportamientos empáticos y altos niveles de interacciones prosociales e interpersonales (Kunzmann et al., 2018; Shoshani et al, 2021; Wong et al., 2021)

Algunas investigaciones han develado que a partir del nacimiento son notables las diferencias en los comportamientos sociales en función del género, incluyendo las predisposiciones empáticas y mostrando que las bebés tienen una tendencia a llorar cuando escuchan otro llanto infantil y cuando conectan visualmente con otros rostros y voces (Fausto-Sterling, 2016; Misailidi \& Tsiara, 2021). Otros investigadores encontraron que las niñas mejoraban la empatía afectiva y cognitiva de manera paralela con la edad, hasta llegar a un periodo de estabilización a los 13 
años (Van der Graaff et al., 2014; Wang et al., 2021); por esta razón las niñas, puntuaban más alto en empatía y solían tener mayor activación de las neuronas espejo (Gazzola et al., 2006). Con respecto a los niños que tenían madres con más conciencia emocional, se encontró que demostraban ser más empáticos, con mayores índices de conocimiento y comprensión emocional (Shoshani et al., 2021), por el contrario, los padres que no estimulaban la expresión emocional podía disminuir el aprendizaje emocional y conducir a niveles bajos de empatía (Ahl \& Dunham, 2019; Taylor et al., 2013). En una investigación colombiana se evidenció que el control del enojo en el contexto social del juego generaba frustración en los niños, refiriéndose a la conducta en menores de dos años, donde el papel de la madre era crucial, entonces, los investigadores afirmaban que el vínculo permite no solo la regulación del comportamiento, sino que también enseña acerca del amor por los otros (Esquivel et al., 2013).

\section{Empatía en el ámbito educativo}

Está claro que la educación no se puede apartar de las emociones, sino que, por el contrario, debe integrarlas y aprender de ellas (Beschorner \& Hall, 2021). Por esta razón, es imperativo que la escuela fortalezca el desarrollo intra e interpersonal de los estudiantes, mejorando los procesos de aprendizaje y las relaciones humanas, claves en el bienestar humano (Herrera \& Buitrago, 2015; Jenkins \& Fredrick, 2017; Steger et al., 2021; Van-Rijn et al., 2019).

Ahora bien, desde la mirada educativa, la empatía se establece como un aspecto importante para la infancia, debido a que los niños en edad escolar, y mediante su desarrollo, pueden comprender las relaciones humanas con más precisión, al igual que la posición del otro con solidaridad, en la medida que se alejan de la etapa del egocentrismo (Fernández-Pinto et al., 2008; Gevaux et al., 2020; Portillo \& Barajas, 2016). Así, una de las relaciones más significativas es la que se establece entre el educador y el niño, ya que este vínculo fomenta una mayor empatía que se devela en las relaciones con otras personas, con la percepción emocional y la reciprocidad establecida a través del lenguaje no verbal (Wee et al., 2021). Es oportuno aclarar que las escuelas atienden a todo el estudiantado con sus individualidades, siendo 
la institución el lugar donde la convivencia y la educación propician el respeto por las diferencias y promueve el desarrollo de capacidades, entonces las habilidades sociales no se desarrollan de manera similar en todas las personas. A este respecto, algunos autores han comentado que, por ejemplo, en el trastorno del espectro autista se evidencia la dificultad en el reconocimiento de las emociones propias y las de los demás, dificultando este hecho las interacciones sociales (Paulus \& Leitherer, 2017; Ruggieri, 2013), dificultad que bloquea la anticipación; en este sentido, Di Martino et al. (2009), Ruggieri (2013), confirman que la baja empatía y la dificultad para anticiparse son índices del espectro autista. También, en el ámbito escolar se ha corroborado disminución específica de la empatía cognitiva en niños con el trastorno del espectro autista (TEA), mientras que en niños con problemas de conducta se disminuye la empatía afectiva (Lee et al., 2019; Schwenck et al., 2012).

No cabe duda que los efectos de los programas de educación emocional, en empatía con niños en edad escolar han sido significativos (Demedardi et al., 2021). En este sentido se han analizado los efectos que ha tenido esta formación, específicamente en niños de los grados de primaria, con la formulación e implementación de actividades que dieron como resultado el aumento de habilidades de empatía (Elenbaas, 2019). De igual manera, Kalliopuska y Tiitinen (1991) realizaron un estudio en el que participaron 61 niños, distribuidos en dos grupos de intervención, resultando una mejoría en los niveles de empatía en la fase post-evaluación en ambos grupos. Otras investigaciones han concordado en sus hallazgos, como el estudio realizado por Pavarino et al. (2005), quienes encontraron que los comportamientos empáticos en el contexto escolar son bastante frecuentes, evidenciado en comportamientos empáticos tales como: ayudar, felicitar y prestar. Otros estudios de diseño experimental, como el de Kalliopuska y Ruokonen (1993) sugieren la implementación de un programa de educación en empatía por medio de la música, que sirve como motivador y tiene incidencia positiva en el desarrollo de habilidades sociales, estos investigadores determinaron que era importante el desarrollo de las mencionadas habilidades sociales en función de la prevención del acoso escolar. 


\section{Conclusiones}

El concepto de empatía ha estado nutrido desde varias perspectivas y áreas del conocimiento, permitiendo que se consolide de forma estructurada y se defina su trabajo y abordaje científico y académico. De esta forma, en la educación infantil se han realizado diversas investigaciones, en el marco nacional e internacional, que han construido bases sólidas para la educación emocional no solo a nivel escolar sino también familiar. El primer lugar donde el niño aprende y construye su 'ser' es la familia. A partir de la relación con sus padres o cuidadores establece vínculos de apego y habilidades emocionales, adecuadas o no. En estos espacios y tiempos, se va desarrollando la capacidad empática, con la edad, por lo cual, su desarrollo en la infancia resulta imperativo, por su impacto posterior en las dimensiones personal, social y escolar.

Las habilidades empáticas se pueden fortalecer en el contexto escolar, gracias a las relaciones que permite la escuela, con los iguales y los maestros, que se gestan en el contexto escolar el desarrollo de varios estudios interesados en indagar por las causas o explicaciones de las situaciones intra e interpersonales visibles en el aula. Con estas investigaciones se han consolidado múltiples programas de formación emocional e intervención psicopedagógica, porque la escuela se constituye en un lugar donde se puede analizar la comunicación, experiencia y preparación de los estudiantes para la vida, no solo en los aspectos académicos sino también en su desarrollo emocional, frente a sí mismo y a los 'otros'.

En este sentido, se hace necesario que en la escuela se establezcan contextos positivos y seguros, que propendan por la felicidad, para lo cual, el desarrollo de la empatía se hace fundamental en la interacción, el aprendizaje, la comunicación, la convivencia y éxito de sus actores. Se sugiere entonces una práctica reflexiva de la educación emocional, tomando como protagonista la experiencia emocional propia, para enriquecer las interrelaciones entre estudiantes, profesor y comunidad educativa, donde los padres de familia asumieran comprometidamente su papel. Por último, a partir de esta revisión sistemática, se pudo evidenciar la influencia de la empatía en el comportamiento de los niños en la escuela, en sus conductas prosociales y disruptivas, situaciones que pueden generalizarse a cualquier espacio 
escolar, sugiriendo la necesidad de establecer ambientes saludables, que consideren los valores, el reconocimiento del otro, el respeto a la diferencia y el desarrollo de la inteligencia emocional. El propósito final es que se propicien conductas prosociales y relaciones interpersonales cálidas; donde la empatía abandere las estrategias y procesos educativos escogidos, desde la formación del profesorado para entenderla, asumirla e impartirla. Como sucede con cualquier otro aspecto formativo, en el caso del desarrollo emocional y empático, lo ideal es comenzar con las edades tempranas, con la primera infancia, de forma que se impacte las etapas de desarrollo futuras, con programas de educación emocional que transversalice el currículo en función de construir una escuela y una sociedad con niños felices y empáticos cambiando el paradigma de la educación.

\section{Referencias}

Ahl, R. E., Dunham, Y. (2019). "Wealth makes many friends": Children expect more giving from resource-rich than resource-poor individuals. Child Development, 90, 524-543. https://doi.org/10.1111/cdev.12922

Angulo, B., Estévez, A., Iriarte, L., Troyas, I. (2019). Empatía y mindfulness como factores mentalizadores intervinientes en las relaciones entre el apego y la sensibilidad interpersonal en mujeres. Ansiedad y Estrés, 25(1), 42-48. https://doi.org/10.1016/j.anyes.2019.04.004

Belacchi, C., Farina, E. (2012). Feeling and thinking of others: Affective and cognitive empathy and emotion comprehension in prosocial/hostile preschoolers. Aggressive behavior, 38(2), 150-165. https://doi.or/10.1002/ab.21415

Beschorner, B., Hall, A. (2021). Building Inclusivity and Empathy Through Writers' Workshop. The Reading Teacher. https://doi.org/10.1002/trtr.1971

Bian, L., Leslie, S.-J., Cimpian, A. (2017). Gender stereotypes about intellectual ability emerge early and influence children's interests. Science, 355, 389-391.

Chlopan, B. E., McCain, M. L., Carbonell, J. L., Hagen, R. L. (1985). Empathy: Review of available measures. Journal of personality and social psychology, 48(3), 635. https://doi.org/10.1037/0022-3514.48.3.635

Chopik, W. J., O'Brien, E., Konrath, S. H. (2016). Differences in empathic concern and perspective taking across 63 countries. Journal of Cross-Cultural Psychology, 48, 23-38. https://doi.org/10.1177/0022022116673910

Cuff, B. M. P., Brown, S. J., Taylor, L., Howat, D. (2016). Empathy: A review of the concept. Emotion Review, 8, 144-153. https://doi.org/10.1177/1754073914558466

De Minzi, M. C. R., Lemos, V., Mesurado, B. (2011). Relaciones entre la percepción que tienen los niños de los estilos de relación y de la empatía de los padres y la conducta prosocial en la niñez media y tardía. Avances en psicología latinoamericana, 29(2), 330-343 
Empatía y educación en la infancia y la niñez: un estado actual de la cuestión

Decety, J., Yoder, K. J. (2016). Empathy and motivation for justice: Cognitive empathy and concern, but not emotional empathy, predict sensitivity to injustice for others. Social Neuroscience, 11, 1-14. https://doi.org/10.1080/17470919.2015.1029593

Decety, J., Meidenbauer, K. L., Cowell, J. M. (2018). The development of cognitive empathy and concern in preschool children: A behavioural neuroscience investigation. Developmental Science, 21(3), https://doi.org/10.1111/desc.12570

Demedardi, M. J., Brechet, C., Gentaz, E., Monnier, C. (2021). Prosocial lying in children between 4 and 11 years of age: The role of emotional understanding and empathy. Journal of Experimental Child Psychology, 203, 105045. https://doi.org/10.1016/i.jecp.2020.105045

Di Martino, A., Ross, K., Uddin, L. Q., Sklar, A. B., Castellanos, F. X., Milham, M. P. (2009). Functional brain correlates of social and nonsocial processes in autism spectrum disorders: an activation likelihood $\begin{array}{llll}\text { estimation } \quad \text { meta-analysis. } & \text { Biological }\end{array}$ https://doi.org/10.1016/i.biopsych.2008.09.022

Díaz, M. S., Vega-Valdés, J. C. F. (2003). Algunos aspectos teórico-conceptuales sobre el análisis documental y el análisis de información. Ciencias de la Información, 34(2), 49-60

Eisenberg, N., Eggum, N. D. (2009). Empathic responding: Sympathy and personal distress. Decety, J., \& Ickes, W. (Eds.). The social neuroscience of empathy. (pp. 71-83) Mit Press.

Eisenberg, N., Strayer, J. (1987). Critical issues in the study of empathy. En Eisenberg, N. \& Strayer, J. (Eds.), Cambridge studies in social and emotional development. Empathy and its development (pp. 3-13). Cambridge University Press.

Elenbaas, L. (2019). Against unfairness: Young children's judgements about merit, equity, and equality. Journal of Experimental Child Psychology, 186, 73-82. https://doi.org/10.1016/j.jecp.2019.05.009

Esquivel, M. F., Cabrero, B. G., Lena, M. M. Y. L., Cruz, A. V. (2013). Regulación materna y esfuerzo de control emocional en niños pequeños. International Journal of Psychological Research, 6(1), 30-40.

Fausto-Sterling, A. (2016). How else can we study sex differences in early infancy? Developmental Psychobiology, 58(1), 5-16. https://doi.org/10.1002/dev.21345

Fernández-Pinto, I., López-Pérez, B., Márquez, M. (2008). Empatía: Medidas, teorías y aplicaciones en revisión. Anales de Psicología, 24(2), 284-298.

Gazzola, V., Aziz-Zadeh, L., Keysers, C. (2006). Empathy and the somatotopic auditory mirror system in humans. Current biology, 16(18), 1824-1829. https://doi.org/10.1016/i.cub.2006.07.072

Gevaux, N. S., Nilsen, E. S., Bobocel, D. R., Gault, S. F. (2020). Children's reactions to inequality: Associations with empathy and parental teaching. Journal of Applied Developmental Psychology, 70, 101189. https://doi.org/10.1016/i.appdev.2020.101189

Herrera, L., Buitrago, R. E. (2015). Educación rural en Boyacá, fortalezas y debilidades desde la perspectiva del profesorado. Praxis \& Saber, 6(12), 169-190.

Javakhishvili, M., Vazsonyi, A. T. (2021). Empathy, self-control, callous-unemotionality, and delinquency: unique and shared developmental antecedents. Child Psychiatry \& Human Development, 1-14. https://doi.org/10.1007/s10578-021-01137-2

Jenkins, L. N., Fredrick, S. S. (2017). Social capital and bystander behavior in bullying: internalizing problems as a barrier to prosocial intervention. Journal of youth and adolescence, 46(4), 757-771. https://doi.org/10.1007/s10964-017-0637-0

Pensamiento y Acción, Tunja (Boyacá-Colombia) - No. 31. Julio - Diciembre 2021. ISSN 0120-1190 -eISSN 2619-3353 DOI: https://doi.org/10.19053/01201190.n31.2021.12569 
Fausto-Andrés Núñez; Leidy-Tatiana Porras-Cruz; Ruth-Nayibe Cárdenas-Soler

Kalliopuska, M., Ruokonen, I. (1993). A study with a follow-up of the effects of music education on holistic development of empathy. Perceptual and Motor Skills, 76(1), 131-137. https://doi.org/10.2466/pms.1993.76.1.131

Kalliopuska, M., Tiitinen, U. (1991). Influence of two developmental programmes on the empathy and prosociability of preschool children. Perceptual and Motor Skills, 72(1), 323-328. https://doi.org/10.2466/pms.1991.72.1.323

Kim, S., Kochanska, G. (2017). Relational antecedents and social implications of the emotion of empathy: Evidence from three studies. Emotion, 17(6), 981-992. https://doi.org/10.1037/emo0000297

Kunzmann, U., Wieck, C., Dietzel, C. (2018). Empathic accuracy: Age differences from adolescence into middle childhood. Cognition and Emotion, 32, 1611-1624. https://doi.org/10.1080/02699931.2018.1433128

Lee, J. J., Hardin, A. E., Parmar, B., Gino, F. (2019). The interpersonal costs of dishonesty: How dishonest behavior reduces individuals' ability to read others' emotions. Journal of Experimental Psychology: General, 148(9), 1557-1574. https://doi.org/10.1037/xge0000639

Levy, J., Yirmiya, K., Goldstein, A., Feldman, R. (2019). Chronic trauma impairs the neural basis of empathy in mothers: relations to parenting and children's empathic abilities. Developmental cognitive neuroscience, 38, 100658. https://doi.org/10.1016/i.dcn.2019.100658

López-Pérez, B., Fernández-Pinto, I., García, F. J. A. (2008). TECA: Test de Empatía Cognitiva y Afectiva. Tea.

Malti, T., Gasser, L., Gutzwiller-Helfenfinger, E. (2010). Children's interpretive understanding, moral judgments, and emotion attributions: Relations to social behaviour. British Journal of Developmental Psychology, 28(2), 275-292. https://doi.org/10.1348/026151009X403838

Miller, S. A. (2009). Children's understanding of second-order mental states. Psychological Bulletin, 135(5), 749773.

Misailidi, P., Tsiara, E. (2021). Conscience and theory of mind in children aged 4 to 7 years. Journal of Experimental Child Psychology, 203, 105007. https://doi.org/10.1016/j.jecp.2020.105007

Montgomery, C. B., Allison, C., Lai, M. C., Cassidy, S., Langdon, P. E., Baron-Cohen, S. (2016). Do adults with high functioning autism or Asperger syndrome differ in empathy and emotion recognition?. Journal of autism and developmental disorders, 46(6), 1931-1940. https://doi.org/10.1007/s10803-016-2698-4

Nickerson, A. B., Mele-Taylor, D. (2014). Empathetic responsiveness, group norms, and prosocial affiliations in bullying roles. School Psychology Quarterly, 29(1), 99. https://doi.org/10.1037/spq0000052

Nielsen, M., Haun, D., Kartner, J., Legare, C. H. (2017). The persistent sampling bias in developmental psychology: A call to action. Journal of Experimental Child Psychology, 162, 31-38. https://doi.org/10.1016/i.jecp.2017.04.017

Nilsen, E., Valcke, A. (2018). Children's sharing with collaborators versus competitors: The impact of theory of mind and executive functioning. Journal of Applied Developmental Psychology, 58, 38-48. https://doi.org/10.1016/i.appdev.2018.08.001

Paulus, M., Leitherer, M. (2017). Preschoolers' social experiences and empathy-based responding relate to their fair resource allocation. Journal of Experimental Child Psychology, 161, 202-210. https://doi.org/10.1016/j.jecp.2017.03.005

Pavarino, M. G., Del Prette, A., Del Prette, Z. A. P. (2005). O desenvolvimento da empatia como prevenção da agressividade na infância. Psico, 36(2), 3.

Portillo, M., Barajas, C. (2016). Teoría de la mente, aceptación entre iguales y auto-percepción social. Apuntes de psicología, 34(1), 47-58.

Pensamiento y Acción, Tunja (Boyacá-Colombia) - No. 31. Julio - Diciembre 2021. ISSN 0120-1190 -eISSN 2619-3353 DOI: https://doi.org/10.19053/01201190.n31.2021.12569 
Empatía y educación en la infancia y la niñez: un estado actual de la cuestión

Reese, E., Bird, A., Tripp, G. (2007). Children's self-esteem and moral self: Links to parent-child conversations regarding emotion. Social Development, 16(3), 460-478. https://doi.org/10.1111/j.14679507.2007.00393.x

Ruggieri, V. L. (2013). Empathy, social cognition and autism spectrum disorders. Revista de neurologia, 56, 1321.

Şahin, M. (2012). An investigation into the efficiency of empathy training program on preventing bullying in primary schools. Children and Youth Services Review, 34(7), 1325-1330. https://doi.org/10.1016/i.childyouth.2012.03.013

Schwenck, C., Mergenthaler, J., Keller, K., Zech, J., Salehi, S., Taurines, R., Freitag, C. M. (2012). Empathy in children with autism and conduct disorder: Group-specific profiles and developmental aspects. Journal of Child Psychology and Psychiatry, 53(6), 651-659. https://doi.org/10.1111/j.1469-7610.2011.02499.x

Shamay-Tsoory, S. G, Aharon-Peretz, J., Perry, D. (2009). Two systems for empathy: a double dissociation between emotional and cognitive empathy in inferior frontal gyrus versus ventromedial prefrontal lesions, Brain, 32, (3), 617-627, https://doi.org/10.1093/brain/awn279

Shamay-Tsoory, S. G., Tormer, R., Goldsher, D., Berger, B. D. Aharon-Peretz, J. (2005). Impaired "Affective Theory of Mind" Is Associated with Right Ventromedial Prefrontal Damage. Cognitive Behavioral Neurology, 18(1), 55-67. https://doi.org/10.1097/01.wnn.0000152228.90129.99

Shoshani, A., Braverman, S., Meirow, G. (2021). Video games and close relations: Attachment and empathy as predictors of children's and adolescents' video game social play and socio-emotional functioning. Computers in Human Behavior, 114, 106578. https://doi.org/10.1016/..chb.2020.106578

Smetana, J. G., Ball, C. L. (2019). Heterogeneity in children's developing moral judgments about different types of harm. Developmental Psychology, 55, 1150-1163. https://doi.org/10.1037/dev0000718

Smetana, J. G., Rote, W. M., Jambon, M., Tasopoulos-Chan, M., Villalobos, M., Comer, J. (2012). Developmental changes and individual differences in young children's moral judgments. Child development, 83(2), 683-696. https://doi.org/10.1111/j.1467-8624.2011.01714.x

Steger, A., Evans, E., Wee, B. (2021). Emotional cartography as a window into children's well-being: Visualizing the felt geographies of place. Emotion, Space and Society, 39, 100772. https://doi.org/10.1016/j.emospa.2021.100772

Taylor, Z. E., Eisenberg, N., Spinrad, T. L., Eggum, N. D., Sulik, M. J. (2013). The relations of ego-resiliency and emotion socialization to the development of empathy and prosocial behavior across early childhood. Emotion, 13(5), 822. https://doi.org/10.1037/a0032894

Van der Graaff, J., Branje, S., De Wied, M., Hawk, S., Van Lier, P., Meeus, W. (2014). Perspective taking and empathic concern in adolescence: gender differences in developmental changes. Developmental psychology, 50(3), 881. https://doi.org/10.1037/a0034325

Van-Rijn, J., Quiñones, E. J., Barham, B. L. (2019). Empathic concern for children and the gender-donations gap. Journal of behavioral and experimental economics, 82, 101462. https://doi.org/10.1016/i.socec.2019.101462

Wang, S., Hu, H., Wang, X., Dong, B., Zhang, T. (2021). The Hidden Danger in Family Environment: The Role of Self-Reported Parenting Style in Cognitive and Affective Empathy Among Offenders. Frontiers in Psychology, 12, 167. https://doi.org/10.3389/fpsyg.2021.588993

Pensamiento y Acción, Tunja (Boyacá-Colombia) - No. 31. Julio - Diciembre 2021. ISSN 0120-1190 -eISSN 2619-3353 DOI: https://doi.org/10.19053/01201190.n31.2021.12569 
Wee, S. J., Kim, S. J., Chung, K., Kim, M. (2021). Development of Children's Perspective-Taking and Empathy through Bullying-Themed Books and Role-Playing. Journal of Research in Childhood Education, 1-16. https://doi.org/10.1080/02568543.2020.1864523

Wispé, L. (1986). The distinction between sympathy and empathy: To call forth a concept, a word is needed. Journal of personality and social psychology, 50(2), 314.

Wong, W. I., Yeung, S. P. (2019). Early gender differences in spatial and social skills and their relations to play and parental socialization in Hong Kong Chinese children. Archives of Sexual Behavior, 48, 1589-1602. https://doi.org/10.1007/s10508-019-1415-8

Wong, W. I., Tsui, W. B. P., Siu, T. S. C. (2021). Empathic accuracy of young boys and girls in ongoing parentchild interactions: Performance and (mis) perception. Journal of Experimental Child Psychology, 203, 105042. https://doi.org/10.1016/i.jecp.2020.105042

Zahn-Waxler, C., Radke-Yarrow, M. (1990). The origins of empathic concern. Motivation and emotion, 14(2), 107-130. https://doi.org/10.1007/BF00991639

Zhang, R., Wang, Z. (2019). The mediating effect of empathy in the quadratic relationship between children's resting RSA and sharing behavior. International Journal of Psychophysiology, 140, 8-14. https://doi.org/10.1016/j.ijpsycho.2019.03.012

Pensamiento y Acción, Tunja (Boyacá-Colombia) - No. 31. Julio - Diciembre 2021. ISSN 0120-1190 -eISSN 2619-3353

DOI: https://doi.org/10.19053/01201190.n31.2021.12569 